HERIOT 原国 WATT

UNIVERSITY

Heriot-Watt University

Research Gateway

\title{
Miniaturized 3-D Cross-Type Receiver for Wirelessly Powered Capsule Endoscopy
}

\section{Citation for published version:}

Khan, S, Pavuluri, SK, Cummins, G \& Desmulliez, MPY 2019, 'Miniaturized 3-D Cross-Type Receiver for Wirelessly Powered Capsule Endoscopy', IEEE Transactions on Microwave Theory and Techniques, vol. 67, no. 5, pp. 1985-1993. https://doi.org/10.1109/TMTT.2019.2893204

\section{Digital Object Identifier (DOI): \\ 10.1109/TMTT.2019.2893204}

\section{Link:}

Link to publication record in Heriot-Watt Research Portal

\section{Document Version:}

Peer reviewed version

\section{Published In:}

IEEE Transactions on Microwave Theory and Techniques

\section{Publisher Rights Statement:}

(C) 2019 IEEE. Personal use is permitted. Permission from IEEE must be obtained for all other uses, in any current or future media, including reprinting/republishing this material for advertising or promotional purposes, creating new collective works, for resale or redistribution to servers or lists, or reuse of any copyrighted component of this work in other works.

\section{General rights}

Copyright for the publications made accessible via Heriot-Watt Research Portal is retained by the author(s) and / or other copyright owners and it is a condition of accessing these publications that users recognise and abide by the legal requirements associated with these rights.

\section{Take down policy}

Heriot-Watt University has made every reasonable effort to ensure that the content in Heriot-Watt Research Portal complies with UK legislation. If you believe that the public display of this file breaches copyright please contact open.access@hw.ac.uk providing details, and we will remove access to the work immediately and investigate your claim. 


\title{
Miniaturized 3-D Cross-Type Receiver for Wirelessly Powered Capsule Endoscopy
}

\author{
Sadeque Reza Khan ${ }^{\circledR}$, Sumanth Kumar Pavuluri, Gerard Cummins ${ }^{\circledR}$, Senior Member, IEEE, \\ and Marc P. Y. Desmulliez ${ }^{(}$, Senior Member, IEEE
}

\begin{abstract}
This paper presents the modeling, implementation, and testing of a cross-type receiver (RX) used for wireless power transfer (WPT) into an endoscopic capsule. A 3-D configuration for the receiver is proposed to increase the wireless link power transfer efficiency (WL-PTE) and enhance its robustness to angular variations of the capsule. The diameter and thickness of the flexible transmitter coils are $200 \mathrm{~mm}$ and $185 \mu \mathrm{m}$, respectively. The diameter of the miniaturized $R X$ is $8 \mathrm{~mm}$, which is appropriate for integration within current commercially available capsules. Experimental results demonstrate a WL-PTE of $1.3 \%$ and complete system efficiency of $0.9 \%$ inside a suitable tissue phantom of the relative permittivity of 300 at $5 \mathrm{MHz}$. A maximum variation of only $8.4 \%$ of the WL-PTE across $90^{\circ}$ orientation of the RX was also measured. The minimum WL-PTE of $1 \%$ is the recorder for $7-\mathrm{cm}$ translational displacement inside the phantom. A simulated specific absorption rate of $0.86 \mathrm{~W} / \mathrm{Kg}$ was calculated, which is well below the limit allowed for medical applications. A maximum temperature variation of $2.2{ }^{\circ} \mathrm{C}$ was also measured for an operating duration of $10 \mathrm{~h}$, which is typical of a capsule journey inside the gastrointestinal tract. The proposed system represents a viable proposition for WPT in capsule endoscopy.
\end{abstract}

Index Terms-Capsule endoscopy (CE), ferrite rod, power transfer efficiency (PTE), specific absorption rate (SAR), 3-D cross-type receiver, wireless power transmission.

\section{INTRODUCTION}

W IRELESS biomedical implantable devices (BIDs) have attracted the attention of many researchers over the past decade for applications ranging from health monitoring [1], disease detection, and prevention [2], and drug delivery [3] to prosthetics [4]. Out of these BIDs, capsule type medical devices are commonly used for the diagnostics of gastrointestinal (GI) disorders due to the relatively painless and rapid earlystage detection capability provided. The increasing clinical usage of capsule endoscopy (CE) [5] requires high-quality and long duration diagnostic yield. However, this yield is limited by the size and weight of the capsule, the power required by the electronics embedded in the capsule, and the

Manuscript received September 26, 2018; revised November 12, 2018; accepted December 27, 2018. This work was supported in part by the U.K. Engineering and Physical Research Council (EPSRC) under the Programme Grant entitled Sonopill (EP/K034537/2) and in part by Heriot-Watt University through the Doctoral Training Programme for International Students. (Corresponding author: Sadeque Reza Khan.)

The authors are with the Institute of Sensors, Signals, and Systems, School of Engineering and Physical Sciences, Heriot-Watt University, EH14 4AS Edinburgh, U.K. (e-mail: srk5@hw.ac.uk).

Color versions of one or more of the figures in this paper are available online at http://ieeexplore.ieee.org.

Digital Object Identifier 10.1109/TMTT.2019.2893204 safety concerns related to both the thermal power dissipated by the capsule and the wireless power absorbed by surrounding tissues. Power requirement is indeed a significant limitation in the development of more diagnostically advanced $\mathrm{CE}$ as power provided by recent batteries have been shown to be proportional to their size [6], [7]. An alternative power source to battery is, therefore, necessary for the next generation of CE.

The portability of wireless power transfer (WPT) technology and its ability to transmit power without wires make it an attractive proposition for sophisticated BIDs, such as pacemakers, retinal implants, and neurostimulators [8]-[10]. Near-field inductive coupling [11] and strong resonant magnetic coupling [12], [13] are the common electromagnetic (EM)-based WPT techniques for BIDs due to their moderate size, the range of transmission, and high-wireless link power transfer efficiency (WL-PTE). Although WPT link using inductive coupling is less susceptible to misalignment between the receiver (RX) and transmitter (TX), the orientation of the capsule inside the GI tract is unpredictable. A single coil, 1-D WPT system cannot, therefore, continuously supply constant power for CE. The size of the RX coil must also be small enough to fit in typical 11-mm-diameter and 26-mm-length capsule [14]. The portability of the TX coil is also a major issue as indicated in previous WPT research [15], [16]. Finally, tissue safety has to be ensured for any successful WPT system in CE.

Several designs that are tolerant to misalignment have been reported in the literature for different WPT applications over the past few years. The strongly coupled magnetic resonance is presented for 3-D WPT coils in [17] and [18]. Two and three orthogonal loops are connected together to mitigate the effect of angular misalignment resulting in an efficiency of more than $60 \%$ [17] and $40 \%$ [18] in the air for the complete range of coil rotation. The diameter of the outer loop of the TX and RX coils in both studies is, however, $100 \mathrm{~mm}$, which is inappropriate for the biomedical implant applications. The frequencies of the proposed systems, 41.5 MHz [17] and $80.2 \mathrm{MHz}$ [18], could also be inappropriate for tissue safety for long-term applications such as CE, and therefore, tissue safety validation is necessary at these frequencies before considering for their application in BIDs. A novel WPT system including six TX and four RX coils is presented in [19]. TX coils are placed at different positions of a $46 \times 24 \times 20 \mathrm{~cm}^{3}$ rodent home cage. The RX coils are positioned around $11 \times 20 \times 22 \mathrm{~mm}^{3}$ box placed on the head of a rat. PTEs of 23.6\%-33.3\% and $6.7 \%-10.1 \%$ in the air have been achieved for a head height 
of 8 and $20 \mathrm{~cm}$, respectively, at $13.56-\mathrm{MHz}$ operation frequency. The configuration proposed where multiple TX coils are positioned on the boundaries of a human patient's room is, however, cumbersome for CE WPT applications. Furthermore, the size of the RX proposed is more than 1.5 times larger than the volume of typical capsules used for CE. A 3-D receiver for $\mathrm{CE}$ is proposed in [20] with three orthogonal coils spread inside the capsule with an outer diameter of $10 \mathrm{~mm}$ and length of $13 \mathrm{~mm}$. The TX coil is installed in a cylinder of $41 \mathrm{~cm}$ in diameter and $30 \mathrm{~cm}$ length surrounding the patient. A system efficiency of $1 \%$ is achieved for an operating frequency of $1 \mathrm{MHz}$ in air. The SAR is recorded as $0.4 \mathrm{~W} / \mathrm{Kg}$. In [6], [21], and [22], a 3-D WPT RX coil for $\mathrm{CE}$ is fabricated on a modified ferrite plate. The size of the $\mathrm{RX}$ coil is $9 \times 5 \mathrm{~mm}^{2}$. The outer diameter of the nonportable Helmholtz TX coil is $30 \mathrm{~cm}$. The RX can deliver $300 \mathrm{~mW}$ of power at $1 \mathrm{MHz}$ of frequency. However, the information on the PTE and SAR is not provided. Another 3-D WPT coil for $\mathrm{CE}$ is presented in [16], where the RX coils are winded around a ferrite slab. The proposed system demonstrates a WL-PTE of around 5\% at the 218-kHz frequency for $400-$ and 11.5-mm-diameter TX and RX coils, respectively. The SAR is recorded as $8 \mathrm{~W} / \mathrm{Kg}$, which is significantly higher to the IEEE guidelines [23]. A miniaturized omnidirectional WPT RX coil similar to [17] and [18] is present in [24] for CE applications. The size of the RX coil is $24 \times 18 \mathrm{~mm}^{2}$ which is not appropriate for a CE capsule. The size of the TX coil is around $78 \times 52 \mathrm{~mm}^{2}$. The system PTE is recorded as less than $5 \%$ for a separation distance of $7 \mathrm{~cm}$ in air and $6.78-\mathrm{MHz}$ operation frequency. This paper does not provide any tissue safety information.

This paper addresses the challenges outlined above by proposing a unique 3-D cross-type RX coil. The coil is analyzed using a set of accurate equations governing the performance of the power transfer link and optimized through a nested multidimensional algorithm presented recently in [25]. A multitransmitter (TX) system is adopted in this paper to improve the WL-PTE for longer separation distance. The TX coils are fabricated on a flexible substrate to demonstrate the portability of the proposed system. The bending effect of the TX coil, which is likely to happen for a WPT system strapped to a patient, is also studied regarding the potential degradation of the WL-PTE performance. Tissue safety is demonstrated by simulating the specific absorption rate (SAR) in the tissue using a human body model found in ANSYS HFSS. Simulations results are validated by measuring the temperature variation at multiple positions inside a phantom, mimicking the dielectric properties of human tissue. Finally, the complete system efficiency (CSE) is analyzed to validate the potential of the proposed system to fulfill the power requirement of the typical commercial capsules.

\section{Modeling AND Optimization}

Fig. 1(a) and (b) shows a schematic of the 3-D crosstype holder of the WPT RX without and with copper wires, respectively. A ferrite rod is used to confine the magnetic flux generated inside the space enclosed by the coil, thereby

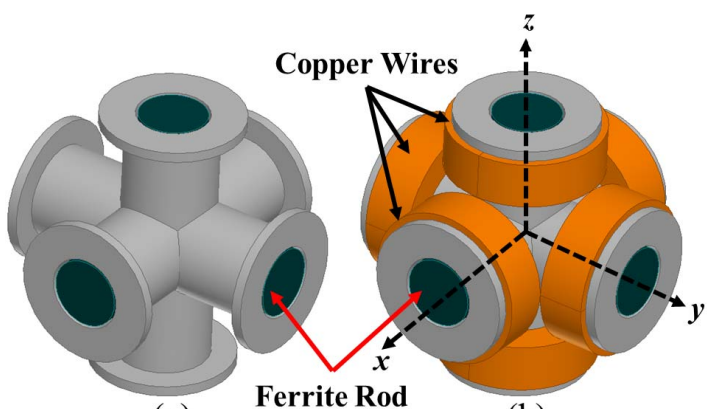

(a)

(b)

Fig. 1. Proposed 3-D cross-type RX coil holder model. (a) Without copper wires. (b) With copper wires.

enhancing the mutual coupling between the TX and RX coils [26].

The self-inductance, $L_{\text {self }}$, of one of the WPT RX coils can be accurately estimated as [27]

$$
\begin{array}{r}
L_{\text {self }}=\sum_{i=1}^{N_{l}} L\left(R_{i}, w\right)+\sum_{i=1}^{N_{l}} \sum_{j=1}^{N_{l}} M\left(R_{i}, R_{j}, 0\right) \times\left(1-\delta_{i j}\right) \\
+\sum_{i=1}^{\left(N_{t}-1\right)} \sum_{j=1}^{N_{l}} \sum_{k=1}^{N_{l} \times\left(N_{t}-i\right)} M\left(R_{j}, R_{k}, d_{T_{i} T_{j}}\right)
\end{array}
$$

where $w$ is the wire diameter, $N_{l}$ is the number of loops, $N_{t}$ is the number of layers, and $d_{T_{i} T_{j}}$ is the distance between the two layers. $R_{i}$ is the radius of the loops. $R_{i}$ and $R_{j}(i \neq j)$ are the radii of two perfectly aligned loops of the same coil and $R_{k}$ is the radius of the loops in different layers. Furthermore, $\delta_{i j}=1$ for $i=j ; \delta_{i j}=0$, otherwise. The $L_{\text {self }}$ of a single circular coil of radius $R$ and the mutual inductance of two perfectly aligned coils with center-to-center distance $d_{i j}$ have been presented in [27].

The continuously varying radius of the loop of a spiral coil is calculated by considering coils as an Archimedean spiral [27]. Accounting for the ferrite effect [26], $L_{\text {self }}$ is rewritten as

$$
L_{F}=L_{\text {self }}\left[2-\frac{R_{F}^{2}}{R^{2}}\left(1-\frac{\mu_{F}}{1+D_{c}\left(\mu_{F}-1\right)}\right)\right]
$$

where $R, R_{F}, \mu_{F}$, and $D_{c}$ are the radii of the receiver coil and the ferrite rod, the relative intrinsic permeability of the ferrite material and the demagnetizing factor, respectively. $D_{C}$ is defined as [26]

$$
D_{c}=\frac{1.51 \zeta^{0.13} R_{F}^{2}}{h_{F}^{2} e^{3}}\left[\ln \left(\frac{1+e}{1-e}\right)-2 e\right] \quad e=\sqrt{1-\left(\frac{2 R_{F}}{h_{F}}\right)^{2}}
$$

where $h_{F}$ is the height of the ferrite rod and $\zeta=h_{F} / 2 R_{F}$.

Consider two current carrying loops, defined as $\mathrm{C} 1$ and $\mathrm{C} 2$ of respective radii $R_{\mathrm{C} 1}$ and $R_{\mathrm{C} 2}$ as shown in Fig. 2.

The expression that describes the mutual inductance of these coils, $M$, for a separation distance between the two loops of $d_{r}$, translational misalignment of $d_{2}$, roll and pitch rotational 


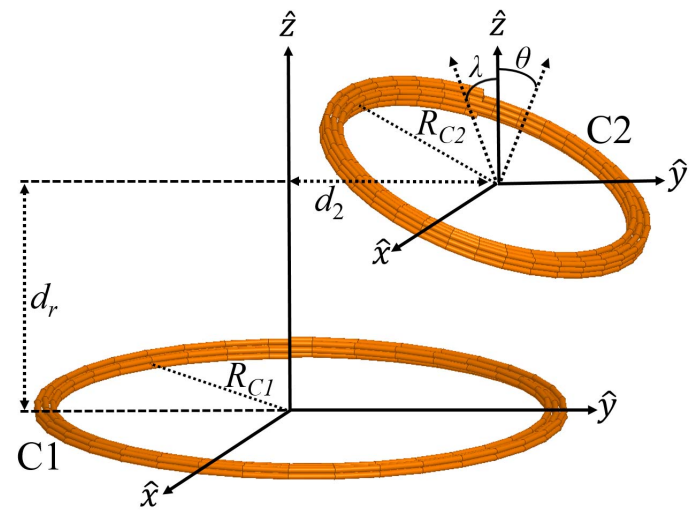

Fig. 2. Translational and angular misalignment of two wirelessly coupled coils.

angle of $\theta$ and $\lambda$, respectively, can be approximated as

$$
\begin{aligned}
M=\frac{\mu \pi R_{C 1}^{2} R_{C 2}^{2} \cos \theta \cos \lambda}{2\left(R_{C 1}^{2}+R_{C 2}^{2}+d_{r}^{2}+d_{2}^{2}\right)^{\frac{3}{2}}} \\
\times\left[\begin{array}{l}
1+\frac{15}{64}\left(\gamma_{a}^{2}+4 \gamma_{b}^{2}\right)+\frac{15}{64}\left(\gamma_{a}^{2}+4 \gamma_{c}^{2}\right) \\
\cos ^{2} \theta \cos ^{2} \lambda+\frac{15}{8} \gamma_{d}^{2}(\sin \theta \cos \lambda \sin \lambda \cos \theta) \\
-\frac{15}{8} \gamma_{c} \gamma_{d} \cos \theta \cos \lambda(\sin \theta \cos \lambda+\sin \lambda \cos \theta) \\
-\frac{3}{2}\left(\delta_{a}-\delta_{b}(\tan \theta+\tan \lambda)\right)
\end{array}\right]
\end{aligned}
$$

where $\mu$ is the permeability of the medium surrounding the coils. In (7), the parameters $\gamma_{a}, \gamma_{b}, \gamma_{c}, \gamma_{d}$, and $\gamma_{e}$ are smaller than unity and defined in [27] alongside $\delta_{a}$ and $\delta_{b}$. Furthermore, the total mutual inductance, $M_{\text {total }}$ accounting for the ferrite effect can be written as [26], [27]

$$
\begin{aligned}
M_{\text {total }}=\sum_{i=1}^{T_{C 1}} \sum_{j=1}^{T_{C 2}} \sum_{k=1}^{L_{C 1}} & \sum_{l=1}^{L_{C 2}} M\left(\begin{array}{l}
R_{C 1: k}, R_{C 2: l}, \\
d_{r(i, j)}, d_{2}, \theta, \lambda
\end{array}\right) \\
\times & {\left[2-\frac{R_{F}^{2}}{R^{2}}\left(1-\frac{\mu_{F}}{1+D_{c}\left(\mu_{F}-1\right)}\right)\right] }
\end{aligned}
$$

where coil $\mathrm{C} 1$ contains $T_{\mathrm{C} 1}$ layers and $L_{\mathrm{C} 1}$ loops, and coils $\mathrm{C} 2$ contains $T_{\mathrm{C} 2}$ layers and $L_{\mathrm{C} 2}$ layers.

The ac resistance with the ferrite effect on the copper wire in the RX is estimated as [26], [28]-[30]

$$
R_{a c}=\frac{\rho l \zeta}{\pi \delta(w-\delta) D_{c}}
$$

where $\rho$ is the resistivity of the copper wire. The length of the wire $l$ is estimated as in [25]. Furthermore, the skin depth, $\delta$ is defined in [16]-[18]. An accurate analysis of the printed spiral coil (PSC) $R_{\text {ac }}$ is presented in [25]. The expression of the parasitic capacitance for copper wire and PSC are described in [31] and [25], respectively.

In this paper, a multi-TX WPT system is considered, in which two TX coils and one coil $\left(\mathrm{RX}_{x}\right)$ of the 3-D RX coil present as shown in Fig. 3. In a single TX coil WPT system, the PTE decreases severely as $d_{r}$ increases. This is not the case if two TX coils are used. In this case, the coupling between TX1 and TX2, $k_{\mathrm{TX} 1, \mathrm{TX} 2}$, is neglected due to the large

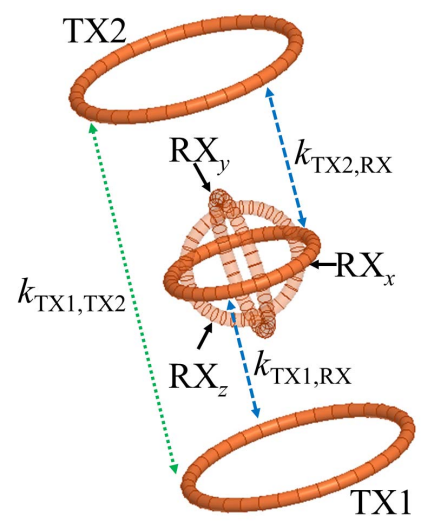

Fig. 3. Two TX and 3-D RX-based WPT system.

separation distance. Furthermore, $\mathrm{RX}_{x}, \mathrm{RX}_{y}$, and $\mathrm{RX}_{z}$ are the coils to receive power from $x$-, $y$-, and $z$-axes, respectively.

The WL-PTE can be defined as the ratio between the total power delivered to the system from the source, $P_{S}$, and the power delivered to the load, $P_{L}$. Based on the energy conservation theory, $P_{S}=P_{\mathrm{TX} 1}+P_{\mathrm{TX} 2}+P_{\mathrm{RX}}+P_{L}$ [32]-[34] for a two TX WPT system where $P_{\mathrm{TX} 1}, P_{\mathrm{TX} 2}$, and $P_{\mathrm{RX}}$ are defined as the powers of the TX1, TX2, and RX coils, respectively. The WL-PTE of the two TX WPT system, $\eta$, using coupled mode theory is

$$
\eta=\frac{P_{L}}{P_{S}}=\frac{1}{2+\frac{\mathrm{Q}_{L}}{\mathrm{Q}_{R X}}\left[2+\frac{1}{F O M D^{2}}\left(1+\frac{\mathrm{Q}_{R X}}{\mathrm{Q}_{L}}\right)^{2}\right]}
$$

where $Q_{\mathrm{RX}}$ and $Q_{L}\left(=2 \pi f L_{\mathrm{RX}} / R_{L}\right)$ [33]-[35] are the quality factor ( $Q$-factor) of the RX coil and load, respectively, where $f, L_{\mathrm{RX}}$, and $R_{L}$ are the operation frequency, selfinductance of the RX coil and load resistor, respectively. The distance-dependent figure of merit (FOMD) is defined as

$$
\mathrm{FOMD}=\sqrt{k_{T X 1, R X}^{2} Q_{T X 1} Q_{R X}+k_{T X 2, R X}^{2} Q_{T X 2} Q_{R X}}
$$

where $Q_{\mathrm{TX} 1}$ and $Q_{\mathrm{TX} 2}$ are $Q$-factor of the TX1 and TX2 coils. Furthermore, $k_{i j}$ is the distance-dependent coupling coefficient between coil $i$ and $j$ [9], [16], [17]. The expression of the optimized load, $R_{L}^{*}$, for the operating frequency, $f$, is

$$
R_{L}^{*}=\frac{2 \pi f L_{R X} \sqrt{1+2 \mathrm{FOMD}^{2}}}{Q_{\mathrm{RX}}} .
$$

The total WL-PTE for the two TX and RX 3-D WPT system is defined as

$$
\eta_{\text {total }}=\eta_{\mathrm{RX}}(x-\text { axis })+\eta_{\mathrm{RX}}(y-\text { axis })+\eta_{\mathrm{RX}}(z-\text { axis }) .
$$

Fig. 4 shows the magnetic field distribution for both single and two TX WPT systems. The RX coil is positioned $10 \mathrm{~cm}$ from the TX coils. Compared to a single TX WPT [Fig. 4(a)], the RX coil in multi-TX WPT is enclosed by higher intensity magnetic field. Therefore, the WL-PTE of a multi-TX WPT is higher compared to a single TX WPT for a large separation distance.

The proposed multi-TX and 3-D RX WPT system is optimized for 5-MHz operation frequency using (1)-(10) to extract the coil parameters such as wire width and number of turns 


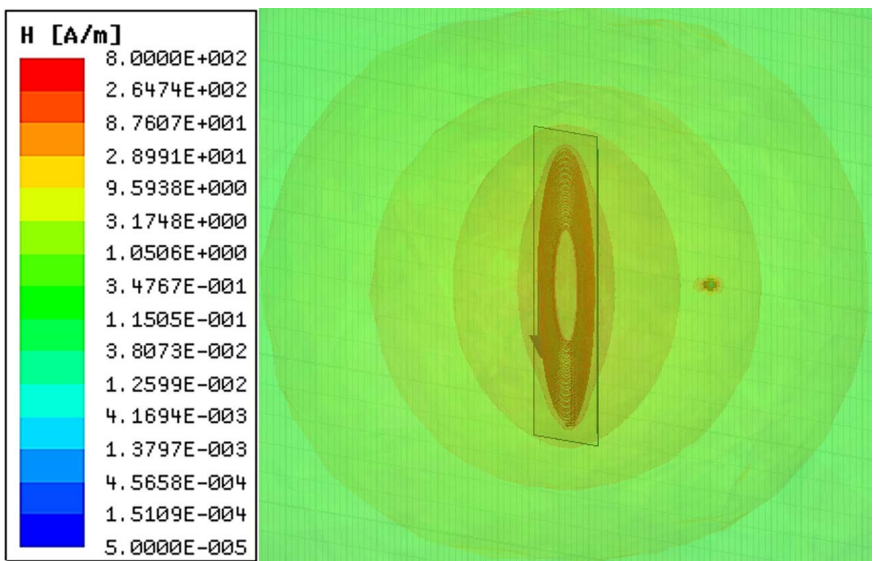

(a)

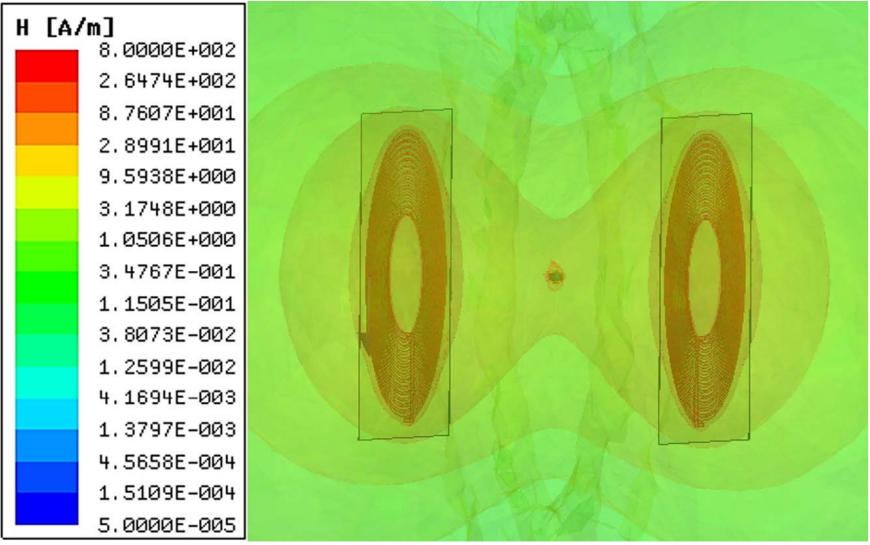

(b)

Fig. 4. Magnetic field distribution. (a) Single TX WPT. (b) Multi-TX WPT.

TABLE I

OPTIMIZED TX AND RX COIL PARAMETERS

\begin{tabular}{ccccccc}
\hline \hline Parameter & $R(\mathrm{~mm})$ & $w(\mathrm{~mm})$ & $s(\mu \mathrm{m})$ & $N$ & $L_{F}(\mu \mathrm{H})$ & $Q$ \\
\hline $\begin{array}{c}\mathrm{TX} \\
\mathrm{RX}(x-, y \text { - } \\
\text { and } z \text {-axis })\end{array}$ & 101 & 2 & 500 & 6 & 15.21 & 224 \\
\hline \hline
\end{tabular}

to improve FOMD and maximum WL-PTE using the nested multidimensional optimization algorithm presented in [25]. This frequency was chosen instead of the ISM band frequency of $6.78 \mathrm{MHz}$ which displays a lower PTE due to the increase in the parasitic components of the TX and RX coils. Moreover, the electronic circuitry of the whole WPT system is cheaper at that frequency. The optimization takes approximately $15 \mathrm{~min}$ to complete in Intel (R) Xeon (R) CPU E5-2640 with a processor speed of $2.5 \mathrm{GHz}$ and $128 \mathrm{~GB}$ of RAM (HP Z820 workstation) computer. The simulation of these types of complex WPT coils requires usually substantial time for commercial EM solver software packages. The optimized load, $R_{L}^{*}$, is calculated as $1.3 \Omega$ for $d_{r}=10 \mathrm{~cm}$. The optimized parameters of the TX and RX coils are listed in Table I, where $s$ and $N$ are the spacing between two copper wires and ta otal number of turns, respectively.

\section{RESUlTS AND DISCUSSION}

Fig. 5(a) shows the 3-D printed coil holders with and without ferrite rod for the proposed RX. Nickel-zinc (NiZn)

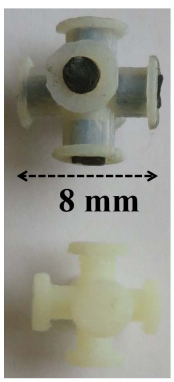

(a)

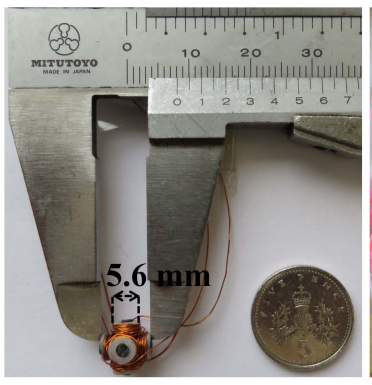

(b)

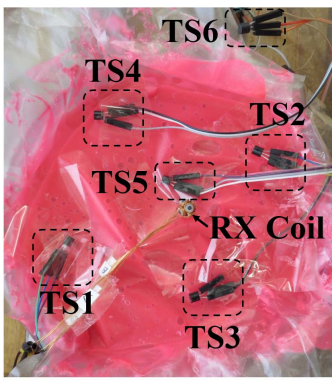

(c)
Fig. 5. (a) 3-D printed coil holders. (b) RX with copper wire. (c) Temperature sensor installation inside the phantom.

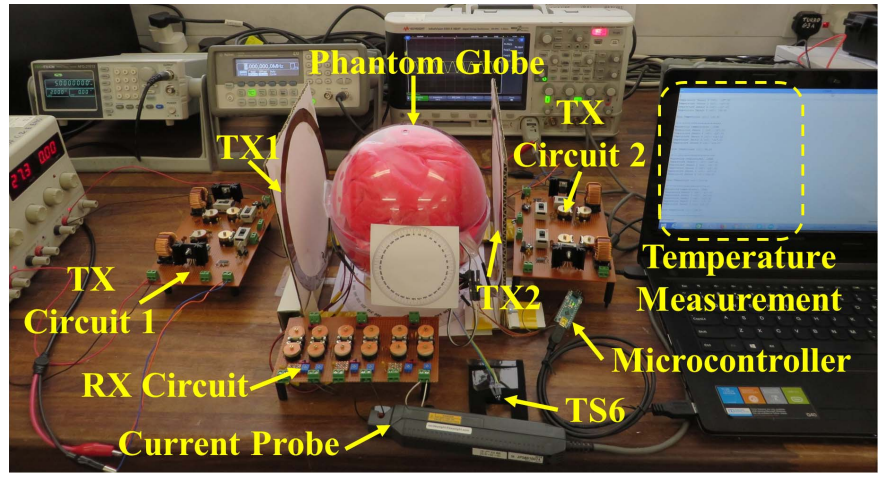

Fig. 6. Measurement setup.

material 61 for the cylindrically shaped ferrite rod of $h_{F}=$ $2.5 \mathrm{~mm}$ and $R_{F}=1 \mathrm{~mm}$ is used to improve the PTE of the 3-D WPT system. Fig. 5(b) shows the RX coil wound with copper wire. The total length of the proposed RX and the diameter of the cylinders onto which the copper wire is wound are 8 and $5.6 \mathrm{~mm}$, respectively, in all three directions. The RX coil is mounted at the center of the solid spherical phantom, as shown in Fig. 5(c). Five temperature sensors (TSs) are inserted approximately equidistantly around the RX coil and TS6 is placed outside the phantom to measure the temperature variation of the phantom due to radio wave radiation with respect to room temperature.

The optimized parameters of WPT coils presented in Table I are used to fabricate the RX and TX coils. The TX coils are manufactured on 150- $\mu$ m-thick DuPont Pyralux AP flexible substrate with a $35-\mu \mathrm{m}$-thick copper cladding. Fig. 6 shows the measurement setup with the multi-TX coils and $18-\mathrm{cm}-$ diameter spherical phantom which is made of two identical hemispheres. The TX coils are $1 \mathrm{~cm}$ away from the phantom. The TX coils are driven by separate TX circuits (class-E amplifier) 1 and 2 as shown in Fig. 6. The phantom has been prepared using hydrophilic organic powder and degassed water [5] to reproduce the EM properties of muscle tissue. A test bench (RX circuit) is prepared using variable capacitors to adjust the resonant condition of the RX coils and optimized load resistors to measure the total WL-PTE (10). The circuit contains also the Schottky diode-based bridge rectifier for each RX coil to measure the CSE. Keysight technologies current probe (1147B) and oscilloscope are used for the accurate measurement of the PTE. Microcontroller Arduino 


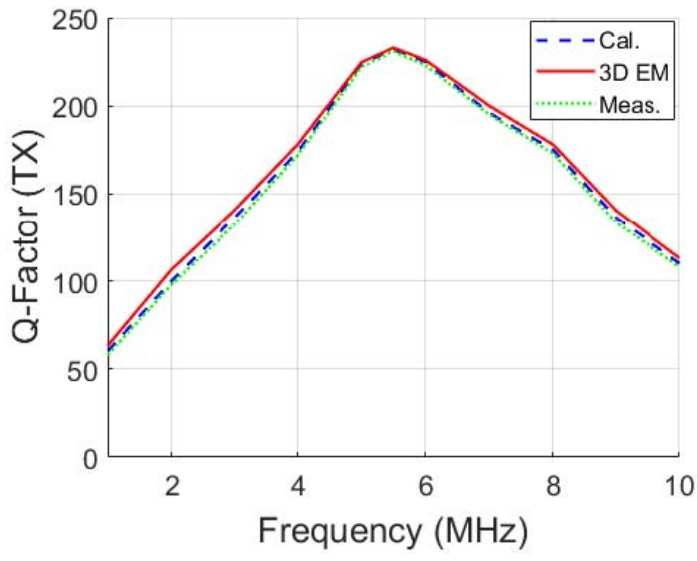

Fig. 7. $Q$-factor of the TX coil versus frequency.

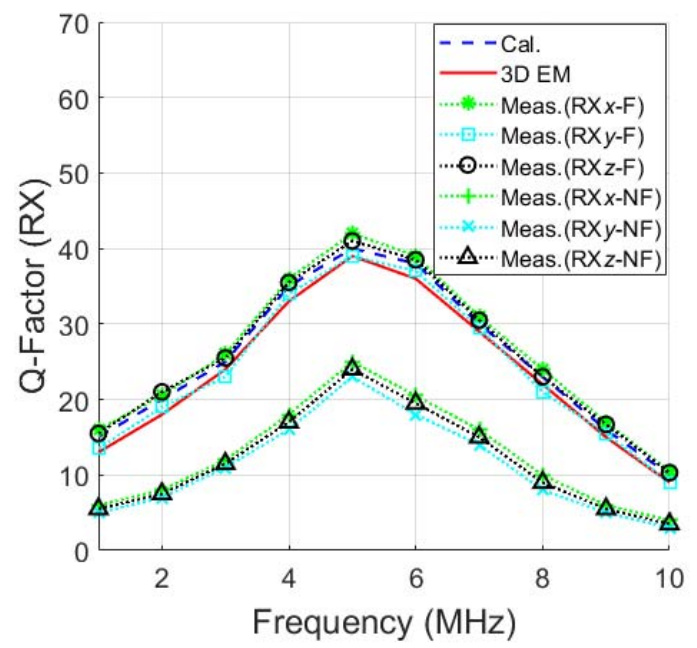

Fig. 8. $Q$-factor of the RX coils versus frequency.

Nano 3.0 is used to measure the temperature variation in a serial communication window.

Figs. 7 and 8 show the variation with frequency of the theoretical, simulated, and measured values of the $Q$-factors for TX and RX coils, respectively, the latter along the three axes. In Fig. 8, the variation of $Q$-factors is provided for RX coils with (-F) and without (-NF) ferrite rods. The impedance analyzer HP 4192A is used to measure the $Q$-factors of the TX and RX coils at different frequencies. Good agreement is observed between the calculated, simulated, and measured results. The highest $Q$-factor is achieved at around $5 \mathrm{MHz}$.

Fig. 9 shows the variation of WL-PTE for TX and RX separation distance ranging from $0.5-20 \mathrm{~cm}$ for singleand multi-TX coils in air and phantom. The single-TX coil demonstrates higher WL-PTE at a low distance compared to multi-TX. However, the WL-PTE drops significantly after $8 \mathrm{~cm}$ and becomes negligible at $20 \mathrm{~cm}$. In contrast, the multi-TX coils maintain promising WL-PTE in the $5-15 \mathrm{~cm}$ distance, which is the separation typically expected between the TX and the RX inserted in the capsule inside the GI tract of a typical human. The measured WL-PTEs at $10 \mathrm{~cm}$ distance with ferrite rod from a single TX coil in air and phantom are $1 \%$ and $0.7 \%$, respectively. These PTEs increase to $1.8 \%$ and $1.3 \%$ for multi-TX coils, respectively. Furthermore, the

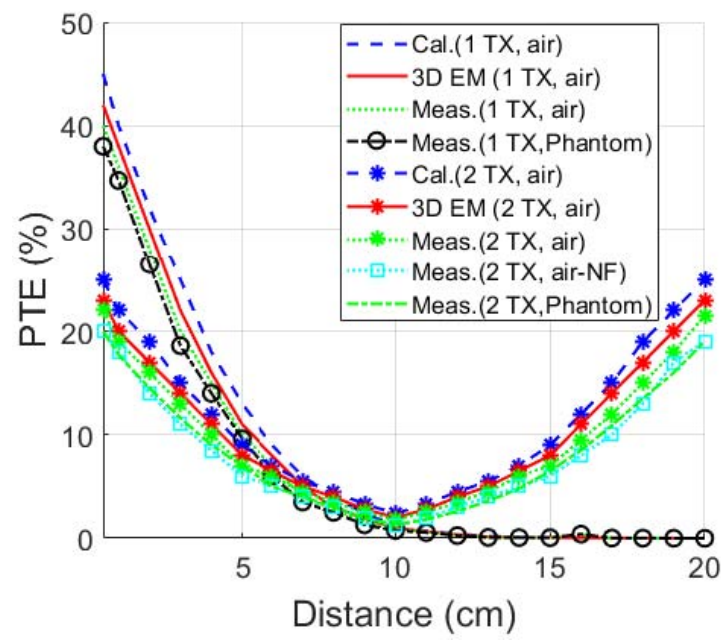

Fig. 9. WL-PTE versus TX and RX separation distance. At $5 \mathrm{MHz}, \varepsilon_{r}=300$, $\sigma=0.59 \mathrm{~S} / \mathrm{m}$, and $\tan \delta=7$ for the phantom

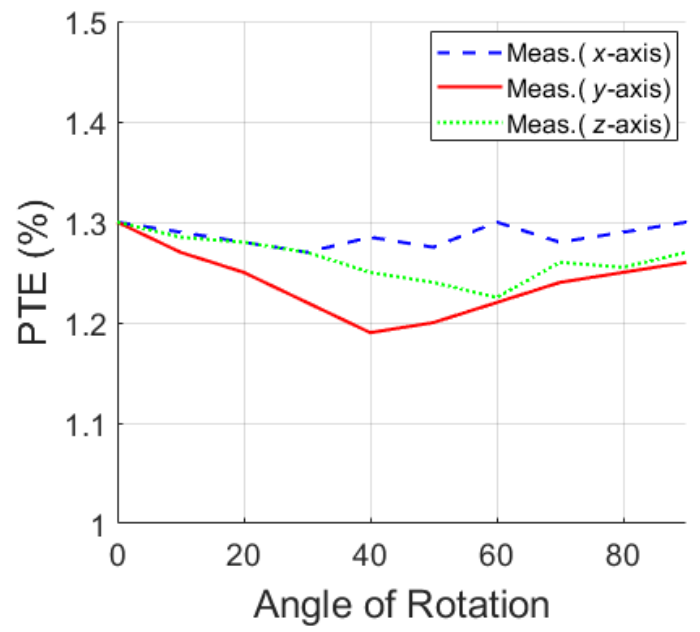

Fig. 10. WL-PTE versus angular rotation for different axes $\left(d_{r}=10 \mathrm{~cm}\right.$ in phantom).

measured WL-PTE for the RX without ferrite rod (NF) and multi-TX at $10 \mathrm{~cm}$ distance in air is $1.2 \%$. Fig. 8 shows the measured $Q$-factor of the RX coils without the ferrite rods are lower compared to the RX coils with ferrite rods. This justifies the reduced WL-PTE (10), for the RX without ferrite rod (NF), as shown in Fig. 9. The relative permittivity, $\varepsilon_{r}$ of the air and phantom is 1 and 300, respectively, at $5 \mathrm{MHz}$. The conductivity, $\sigma$, and loss tangent, $\tan \delta$ of the phantom are $0.59 \mathrm{~S} / \mathrm{m}$ and 7 , respectively.

Fig. 10 shows the total measured WL-PTE (10) with the angular rotation $\left(0^{\circ}-90^{\circ}\right)$ of the phantom globe in the $x-, y$-, and $z$-axes. The proposed 3-D WPT system maintains total WL-PTE at over $1 \%$ for a complete rotation of the RX inside phantom with a maximum variation of $2.3 \%, 8.4 \%$, and $5.8 \%$ in the $x-, y$-, and $z$-axes, respectively. The 3 -D cross-type RX shows great robustness to angular movement of the capsule containing it. Different variations of the WL-PTE along the three axes have been found due to the difference of $Q$-factors in the three axes of the hand wound $\mathrm{RX}$ coil. The $Q$-factor of the manufactured $\mathrm{RX}_{x}, \mathrm{RX}_{y}$, and $\mathrm{RX}_{z}$ coils were indeed 


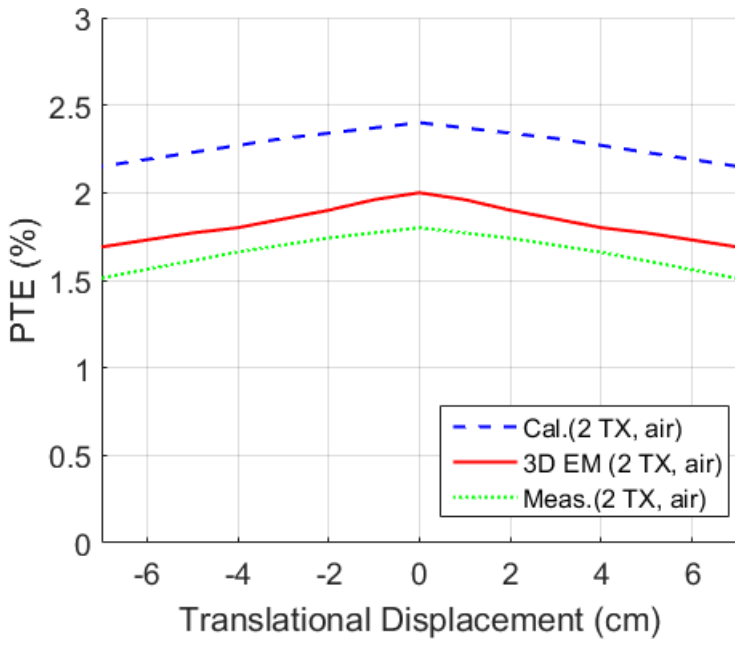

Fig. 11. WL-PTE versus translational displacement (left to right) for $\mathrm{RX}_{x}$ $\left(d_{r}=10 \mathrm{~cm}\right)$

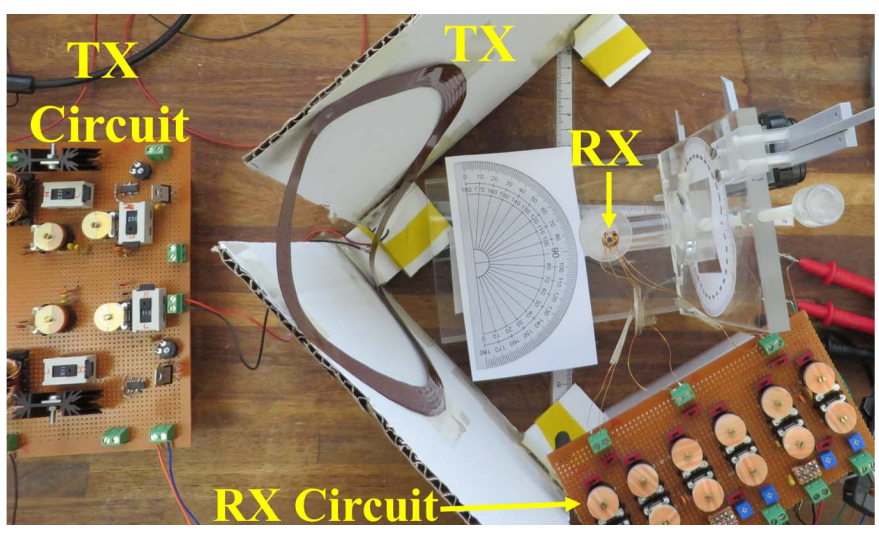

Fig. 12. Flexible TX coil bending effect measurement.

measured at 42,39 , and 41 , respectively, compared to the simulated RX coil $(Q-$ factor $=40)$, where the number of turns are uniform in all the three axes of the RX coil. Therefore, the variation of the total WL-PTE is lower in the rotation of the $x$-axis compared to the rotation in the $y$ - and $z$-axes.

In Fig. 11, the effect of translational displacement on the WL-PTE is demonstrated for $\mathrm{RX}_{x}$ for multi-TX coils. The $\mathrm{RX}_{x}$ is kept at $d_{r}=10 \mathrm{~cm}$ and $0^{\circ}$ rotational angle with the TX coils. The measurement is conducted for $-7 \mathrm{~cm}$ (left) to $+7 \mathrm{~cm}$ (right) translational distances. The minimum WL-PTE recorded for calculation, simulation, and measurement in the air are $2.15 \%, 1.69 \%$, and $1.51 \%$, respectively. The measured minimum WL-PTE of $1 \%$ is achieved in the phantom case for $7 \mathrm{~cm}$ of translational displacement. Furthermore, $\mathrm{RX}_{y}$ and $\mathrm{RX}_{z}$ show similar WL-PTE values for the same range translational displacement.

Fig. 12 shows the bending setup of the TX coil. Such a bending situation would occur when TXs are strapped onto a patient. Fig. 13 shows the results of the bending effect on the WL-PTE as measured in air. The flexible TX coil is placed $12 \mathrm{~cm}$ away from the RX and bended from $0^{\circ}$ to $90^{\circ}$. Fig. 13 shows an increase in the WL-PTE due to $90^{\circ}$ bending of TX coil by $58 \%$. The bending of TX coil concentrates

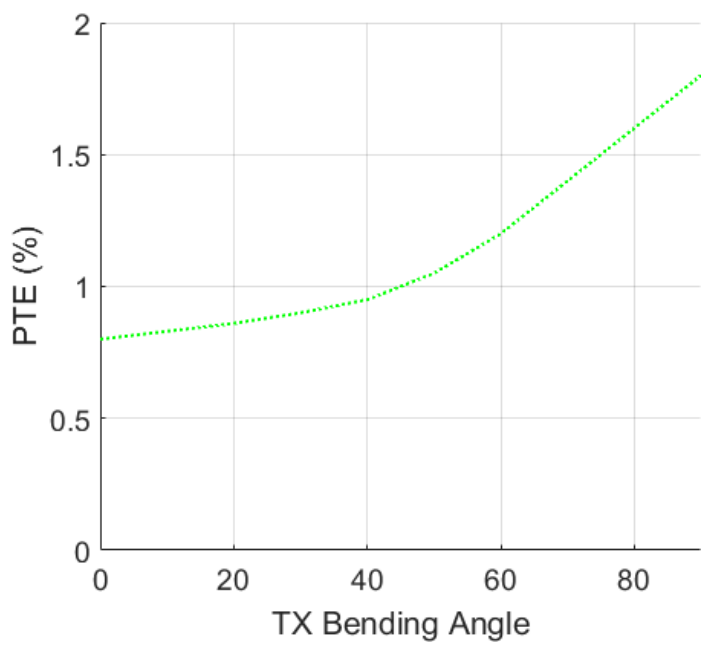

Fig. 13. WL-PTE versus TX coil bending angle $\left(d_{r}=12 \mathrm{~cm}\right.$ in air).

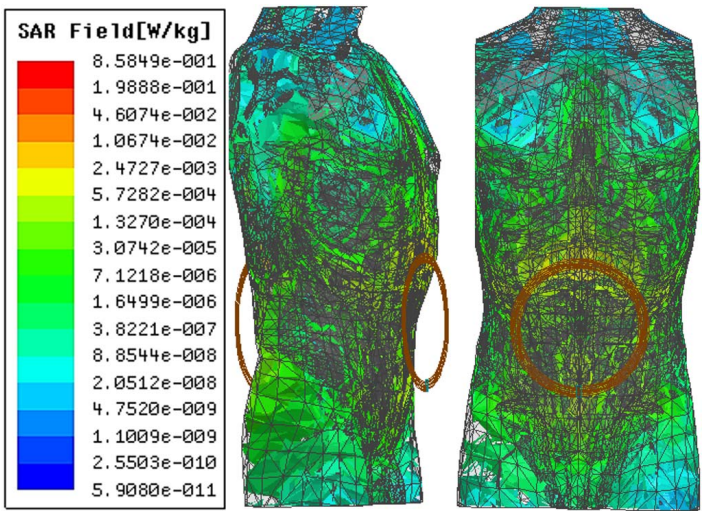

Fig. 14. SAR simulation in the human model in ANSYS HFSS.

the magnetic field toward the RX and reduces the relative separation distance.

Fig. 14 shows the SAR simulation results using the ANSYS HFSS human model. At $f=5 \mathrm{MHz}$ and $10 \mathrm{~W}$ of input power, the SAR value of $0.86 \mathrm{~W} / \mathrm{kg}$ is lower than the IEEE standard of $2 \mathrm{~W} / \mathrm{Kg}$ for $10 \mathrm{~g}$ of tissue [23].

Safety of the tissue was also verified by measuring the variation of temperature, shown in Fig. 15, in the phantom using multiple TS's for a duration of $10 \mathrm{~h}$, which is the typical transit time of a capsule along the GI track.

The temperature in TS5 is recorded more than other TSs as it is mounted close to RX coil. In Fig. 14, SAR of $0.86 \mathrm{~W} / \mathrm{kg}$ is noted in the region near TX coils. TS1 and TS2 demonstrate a similar effect as they are located close to and in parallel with the TX coils. In Fig. 15, after $10 \mathrm{~h}$, the measured maximum temperature variation is measured between $1.5{ }^{\circ} \mathrm{C}$ and $2.2{ }^{\circ} \mathrm{C}$ with respect to room temperature (TS6). TS3 and TS4 are located away and opposite side of the TX coils. Therefore, a lower variation of temperature is noticed in TS3 and TS4 compared to TS1 and TS2.

A new figure of merit, FOM, is proposed to evaluate and compare the performance of the various WPT techniques. This figure of merit can be written as

$$
\mathrm{FOM}=\frac{\text { PTE } \times \text { separation distance } \times \text { axes }}{\text { TX diameter } \times \text { RX size } \times f \times \mathrm{SAR}^{2}} .
$$


TABLE II

Performance COMPARISON

\begin{tabular}{|c|c|c|c|c|c|}
\hline Parameter & [16] & [36] & [38] & [37] & This work \\
\hline TX Diameter (mm) & 400 & 220 & 100 & 23.5 & 200 \\
\hline $\mathrm{RX}$ size $\left(\mathrm{mm}^{2}\right)$ & $11.5 \times 11.5$ & $9 \times 6$ & $15 \times 7$ & $15 \times 7$ & $8 \times 8$ \\
\hline Axes (-D) & 3 & 1 & 3 & 1 & 3 \\
\hline Distance $(\mathrm{cm})$ & 20 & 7 & 5 & 5 & 10 \\
\hline WL-PTE (\%) & 5.02 & 0.7 & 19 & 1.21 & 1.3 \\
\hline$f(\mathrm{MHz})$ & 0.218 & 16.47 & 0.750 & 433.9 & 5 \\
\hline SAR $(\mathrm{W} / \mathrm{Kg})$ & 8 & 1.74 & - & 2.54 & 0.86 \\
\hline Test environment & Air & Pig muscle & Air & Duck intestine & Muscle phantom \\
\hline$F O M\left(\times 10^{03}\right)(11)$ & 4.08 & 0.0824 & - & 0.009 & 8.23 \\
\hline
\end{tabular}

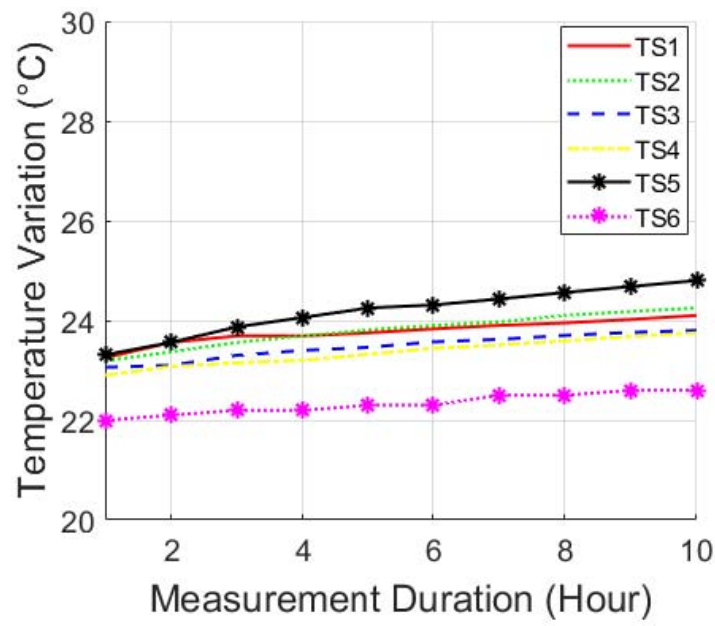

Fig. 15. Variation of temperature in complete CE duration.

In (11), a higher FOM represents better WPT performance. Higher WL-PTE and the large separation distance between the TX and RX coils are desirable in the WPT systems. Furthermore, the term "axes" in (11) is representing the many directions where the RX can receive power. In our case, this number would be 3 . In the applications where the RX is significantly prone to misalignment, the $\mathrm{RX}$ must capable to receive power from all the directions ( $x$-, $y$-, and $z$-axes). Furthermore, the reduced diameter and size of TX and RX coils, respectively, is an important parameter for BIDs. Higher frequency leads to the reduction of the tissue relative permittivity; therefore, can increase the tissue absorption. The square of SAR highlights the importance of tissue safety. The performance of the proposed 3-D WPT system is compared with other recent WPT systems for CE application as listed in Table II. In [16], the proposed 3-D WPT system can achieve WL-PTE of around 5\% in air at the $218 \mathrm{-kHz}$ frequency for a nonportable TX coil. However, the reported SAR is dangerously higher than the recommended IEEE guidelines [23]. Furthermore, the WPT systems for CE presented in [36] and [37] are sensitive to misalignment, not portable 38], or display a high SAR [37]. In [38], the diameter of the cylindrical TX coil $(100 \mathrm{~mm})$ is significantly lower and inappropriate to fit around an average human body.

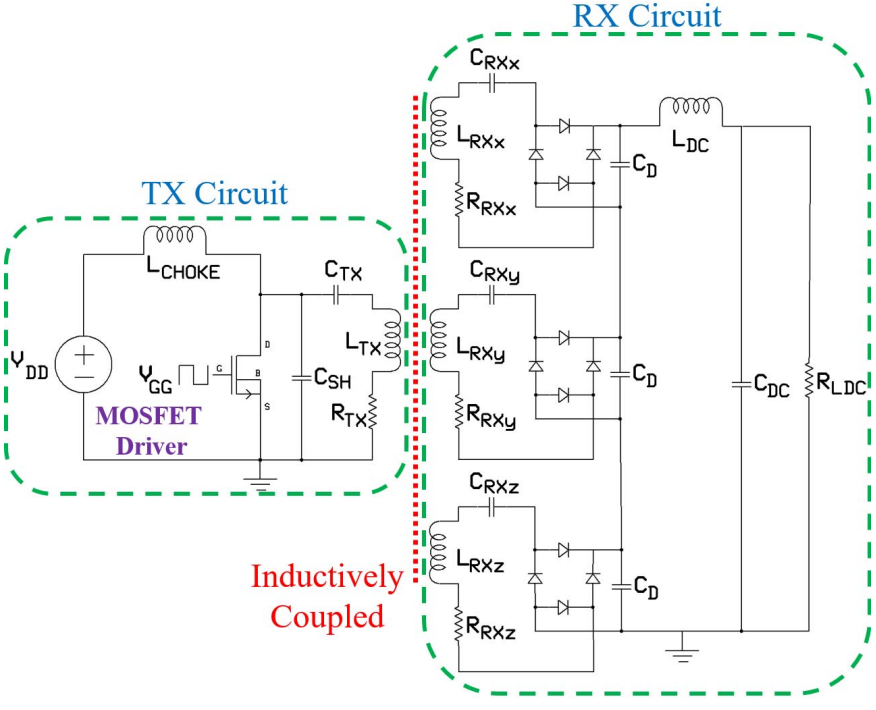

Fig. 16. Schematic of the complete WPT system.

Furthermore, the proposed 3-D rectangular shaped RX coil and its electronic circuitry occupy a significant portion of the capsule volume leaving, thereby little room for other necessary circuits. Compared to the other research works presented in Table II, the proposed system offers a portable and flexible multi-TX coils which can be fit easily around an average human body. The size of the proposed 3-D WPT RX coil is also ideal for CE applications and offers enough space inside the capsule for other important circuits. Furthermore, the efficiency is measured for higher separation distance than [36], [37]. Tissue safety is confirmed by calculation of the SAR and demonstrated experimentally through measurement of the temperature variation [39] inside the phantom. Finally, the calculated FOM confirms the better performance of the proposed 3-D WPT system compared to other recently published research works for CE.

Fig. 16 shows the complete WPT system implemented in this paper. One TX circuit of the multi-TX WPT system is demonstrated, where both TX coils are powered with separate TX circuits. A dc-fed energy injection single-ended class-E power amplifier (PA) is used to implement the TX circuit section [40]. The amplifier is designed using closed- 


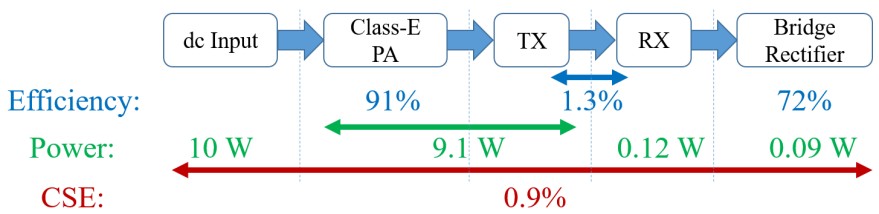

Fig. 17. Efficiency analysis of the complete WPT system.

form equations of the subnominal model indicated in [41] for 5-MHz operating frequency. IRF640 N-channel MOSFET with a breakdown voltage of $200 \mathrm{~V}$ and $R_{\mathrm{DS}}=0.18 \Omega$ is used as the switch with EL7457 noninverting MOSFET driver. Adjustable capacitors are used as shunt capacitor, $C_{\mathrm{SH}}=600 \mathrm{pF}$ and a series resonant capacitor, $C_{\mathrm{TX}}=67 \mathrm{pF}$. $C_{\mathrm{SH}}$ also includes the parasitic capacitance of the MOSFET whose value is $400 \mathrm{pF}$ obtained from the technical datasheet. An RFC inductor, $L_{\mathrm{CHOKE}}=10 \mathrm{mH}$, is utilized to stabilize the output current of the TX circuit. The measured efficiency of the designed $\mathrm{PA}$ is $91 \%$ at $5 \mathrm{MHz}$, where $L_{\mathrm{TX}}$ and $R_{\mathrm{TX}}$ are the inductance and resistance of the TX coil, respectively. In Fig. 16, the inductively coupled RX circuit consists of three $L C$ series resonant circuits formed by three RX coils and their resonant capacitors, $C_{\mathrm{RX}} \cdot L_{\mathrm{RX}}$ and $R_{\mathrm{RX}}$ are the inductance and resistance of the cross-type 3-D RX. Three full-wave bridge rectifiers are connected in a series rectification topology. Schottky diode DB2S20500L with surface mount package $(1.6 \mathrm{~mm} \times 0.8 \mathrm{~mm} \times 0.6 \mathrm{~mm})$ is used to implement the bridge rectifiers. The three outputs are then dc-combined and share the same dc filter where $C_{D}=100 \mathrm{pF}, L_{\mathrm{DC}}=100 \mathrm{nH}$ [42], and $C_{\mathrm{DC}}=1 \mu \mathrm{F}$. The value of the load resistor, $R_{\mathrm{LDC}}$, is considered the same as the optimized load for the WPT link to measure the efficiency of the complete system.

The CSE of the proposed WPT system is $0.9 \%$ for the PA, multi-TX WPT link, and Schottky diode-based bridge rectifier whose respective efficiencies are 91\%, 1.3\%, and $72 \%$, as shown in Fig. 17. The final output power is $90 \mathrm{~mW}$ for a total input power of $10 \mathrm{~W}$, where $5-\mathrm{W}$ input power is supplied in each TX circuit. The current commercial CE typically requires around $30 \mathrm{~mW}$ of power [43], [44]. In the worst-cases of angular and translational misalignment of the proposed WPT link, the achievable output power is still $70 \mathrm{~mW}$. Therefore, the proposed WPT system including multiTX coils and 3-D cross-type RX coil can support current CE even for worst case misalignment conditions. Furthermore, it also has enough power to support other sensor and actuator modalities. It is possible to improve the CSE to $1.03 \%$ by utilizing application specific integrate chip-based high-efficiency (more than 87\%) rectifier circuits [45], [46].

\section{CONCLUSION}

In this paper, a unique 3-D cross-type RX coil and multiTX 3-D WPT system are presented for CE applications. The proposed 8-mm-diameter RX coil can easily fit into a commercial endoscopic capsule. The measured results demonstrate promising PTE at larger separation distances and for complete capsule rotation inside phantom. Total WL-PTE of over $1 \%$ with a CSE of $0.9 \%$ has been measured in human tissue-mimicking phantom material for a separation distance of $10 \mathrm{~cm}$ between the TX and RX. A maximum WL-PTE variation of $8.4 \%$ is measured across $90^{\circ}$ orientation of the $\mathrm{RX}$ in all three axes. In addition, the worst case WL-PTE of $1 \%$ is measured for the maximum translational distance of $7 \mathrm{~cm}$. The beneficial effect of the TX coil flexibility on PTE in terms of bending effect is also presented. The flexible TX coils have been designed to enhance portability and patient's comfort. The tissue safety is verified by SAR simulation and temperature variation measurement inside phantom. In an operating duration of $10 \mathrm{~h}$, the maximum variation of the temperature is recorded as $2.2{ }^{\circ} \mathrm{C}$. The proposed 3-D WPT system is therefore demonstrated to be a potential candidate for an alternative power source for CE.

\section{REFERENCES}

[1] C. Gong, D. Liu, Z. Miao, and M. Li, "A magnetic-balanced inductive link for the simultaneous uplink data and power telemetry," Sensors, vol. 17, no. 8, p. 1768, Jul. 2017.

[2] V. K. Sharma, "The future is wireless: Advances in wireless diagnostic and therapeutic technologies in gastroenterology," Gastroenterology, vol. 137, no. 2, pp. 434-439, Aug. 2009.

[3] T. Maleki, N. Cao, S. H. Song, C. Kao, S.-C. Ko, and B. Ziaie, "An ultrasonically powered implantable micro-oxygen generator (IMOG)," IEEE Trans. Biomed. Eng., vol. 58, no. 11, pp. 3104-3111, Nov. 2011.

[4] R. A. Bercich et al., "Enhancing the versatility of wireless biopotential acquisition for myoelectric prosthetic control," J. Neural Eng., vol. 13, no. 4, p. 046012, Jun. 2016.

[5] J. Faerber et al., "In vivo characterization of a wireless telemetry module for a capsule endoscopy system utilizing a conformal antenna," IEEE Trans. Biomed. Circuits Syst., vol. 12, no. 1, pp. 95-105, Feb. 2017.

[6] R. Carta, M. Sfakiotakis, N. Pateromichelakis, J. Thoné, D. P. Tsakiris, and R. Puers, "A multi-coil inductive powering system for an endoscopic capsule with vibratory actuation," Sens. Actuators A, Phys., vol. 172, no. 1, pp. 253-258, Dec. 2011.

[7] Z. Bingquan, Y. Guozheng, J. Zhiwei, and S. Yu, "Portable wireless power transmission system of a video capsule endoscopy: Design and realization," in Proc. Int. Conf. Biomed. Eng. Biotechnol., May 2012, pp. $409-412$.

[8] K. M. Silay, C. Dehollain, and M. Declercq, "A closed-loop remote powering link for wireless cortical implants," IEEE Sensors J., vol. 13, no. 9, pp. 3226-3235, Sep. 2013.

[9] X. Zhang, S. L. Ho, and W. N. Fu, "A hybrid optimal design strategy of wireless magnetic-resonant charger for deep brain stimulation devices," IEEE Trans. Magn., vol. 49, no. 5, pp. 2145-2148, May 2013.

[10] R. Jegadeesan, S. Nag, K. Agarwal, N. V. Thakor, and Y.-X. Guo, "Enabling wireless powering and telemetry for peripheral nerve implants," IEEE J. Biomed. Health Informat., vol. 19, no. 3, pp. 958-970, May 2015.

[11] U.-M. Jow and M. Ghovanloo, "Design and optimization of printed spiral coils for efficient transcutaneous inductive power transmission," IEEE Trans. Biomed. Circuits Syst., vol. 1, no. 3, pp. 193-202, Sep. 2007.

[12] S. R. Khan and G. Choi, "Optimization of planar strongly coupled wireless power transfer system for biomedical applications," Microw. Opt. Technol. Lett., vol. 58, no. 8, pp. 1861-1866, Aug. 2016.

[13] S. R. Khan and G. Choi, "Analysis and optimization of four-coil planar magnetically coupled printed spiral resonators," Sensors, vol. 16, no. 8, p. 1219, Aug. 2016.

[14] J. Wang, M. Leach, E. G. Lim, Z. Wang, R. Pei, and Y. Huang, "An implantable and conformal antenna for wireless capsule endoscopy," IEEE Antennas Wireless Propag. Lett., vol. 17, no. 7, pp. 1153-1157, Jul. 2018.

[15] W. Xin, G. Yan, and W. Wang, "Study of a wireless power transmission system for an active capsule endoscope," Int. J. Med. Robot. Comput. Assist. Surg., vol. 6, no. 1, pp. 113-122, Mar. 2010.

[16] J. Zhiwei, Y. Guozheng, Jiangpingping, W. Zhiwu, and L. Hua, "Efficiency optimization of wireless power transmission systems for active capsule endoscopes," Physiol. Meas., vol. 32, no. 10, pp. 1561-1573, Aug. 2011.

[17] O. Jonah, S. V. Georgakopoulos, and M. M. Tentzeris, "Orientation insensitive power transfer by magnetic resonance for mobile devices," in Proc. IEEE Wireless Power Transf. (WPT), May 2013, pp. 5-8. 
[18] D. Liu, H. Hu, and S. V. Georgakopoulos, "Misalignment sensitivity of strongly coupled wireless power transfer systems," IEEE Trans. Power Electron., vol. 32, no. 7, pp. 5509-5519, Jul. 2016.

[19] Y. Jia et al., "Position and orientation insensitive wireless power transmission for enerCage-homecage system," IEEE Trans. Biomed. Eng., vol. 64 , no. 10 , pp. $2439-2449$, Oct. 2017.

[20] B. Lenaerts and R. Puers, "An inductive power link for a wireless endoscope," Biosensors Bioelectron., vol. 22, no. 7, pp. 1390-1395, Feb. 2007.

[21] R. Carta, J. Thoné, and R. Puers, "A wireless power supply system for robotic capsular endoscopes," Sens. Actuators A, Phys., vol. 162, no. 2, pp. 177-183, Aug. 2010.

[22] R. Carta and R. Puers, "Wireless power and data transmission for robotic capsule endoscopes," in Proc. 18th IEEE Symp. Commun. Veh. Technol. Benelux (SCVT), Nov. 2011, pp. 1-6.

[23] K. N. Bocan, M. H. Mickle, and E. Sejdić, "Multi-disciplinary challenges in tissue modeling for wireless electromagnetic powering: A review," IEEE Sensors J., vol. 17, no. 20, pp. 6498-6509, Oct. 2017.

[24] A. Pacini, F. Benassi, D. Masotti, and A. Costanzo, "Design of a RF-to-DC link for in-body IR-WPT with a capsule-shaped rotationinsensitive receiver," in IEEE MTT-S Int. Microw. Symp. Dig., Jun. 2018, pp. 1289-1292.

[25] S. R. Khan, S. K. Pavuluri, and M. P. Y. Desmulliez, "New analytical model for the characterisation of printed spiral coils for wireless power transfer," in Proc. IEEE 12th Eur. Conf. Antennas Propag. (EuCAP), Jan. 2018, p. 563.

[26] P. T. Theilmann and P. M. Asbeck, "An analytical model for inductively coupled implantable biomedical devices with ferrite rods," IEEE Trans. Biomed. Circuits Syst., vol. 3, no. 1, pp. 43-52, Feb. 2009.

[27] S. R. Khan, S. K. Pavuluri, and M. P. Y. Desmulliez, "Accurate modeling of coil inductance for near-field wireless power transfer," IEEE Trans. Microw. Theory Techn., vol. 66, no. 9, pp. 4158-4169, Sep. 2018.

[28] M. Kiani and M. Ghovanloo, "A figure-of-merit for designing highperformance inductive power transmission links," IEEE Trans. Ind. Electron., vol. 60, no. 11, pp. 5292-5305, Nov. 2013.

[29] M. Kiani, U.-M. Jow, and M. Ghovanloo, "Design and optimization of a 3-Coil inductive link for efficient wireless power transmission," IEEE Trans. Biomed. Circuits Syst., vol. 5, no. 6, pp. 579-591, Dec. 2011.

[30] H. A. Wheeler, "Formulas for the skin effect," Proc. IRE, vol. 30, no. 9, pp. 299-311, Sep. 1942.

[31] Z. Yang, W. Liu, and E. Basham, "Inductor modeling in wireless links for implantable electronics," IEEE Trans. Magn., vol. 43, no. 10, pp. 3851-3860, Oct. 2007.

[32] M. Kiani and M. Ghovanloo, "The circuit theory behind coupled-mode magnetic resonance-based wireless power transmission," IEEE Trans. Circuits Syst. I, Reg. Papers, vol. 59, no. 9, pp. 2065-2074, Sep. 2012.

[33] A. Kurs, A. Karalis, R. Moffatt, J. D. Joannopoulos, P. Fisher, and M. Soljačić, "Wireless power transfer via strongly coupled magnetic resonances," Science, vol. 317, no. 5834, pp. 83-86, Jul. 2007.

[34] A. Karalis, J. D. Joannopoulos, and M. Soljačić, "Efficient wireless non-radiative mid-range energy transfer," Ann. Phys., vol. 323, no. 1, pp. 34-48, Jan. 2008.

[35] C. Moorey, W. Holderbaum, and B. Potter, "Investigation of highefficiency wireless power transfer criteria of resonantly-coupled loops and dipoles through analysis of the figure of merit," Energies, vol. 8 , no. 10 , pp. 11342-11362, Sep. 2015.

[36] K. Na, H. Jang, H. Ma, and F. Bien, "Tracking optimal efficiency of magnetic resonance wireless power transfer system for biomedical capsule endoscopy," IEEE Trans. Microw. Theory Techn., vol. 63, no. 1, pp. 295-303, Jan. 2015.

[37] K. Ding, Y. Yu, H. Lin, and J. Xie, "Wireless power transfer at sub-GHz frequency for capsule endoscope," Prog. Electromagn. Res. C, vol. 66, pp. 55-61, Jul. 2016

[38] I. Ghotbi, M. Najjarzadegan, S. J. Ashtiani, O. Shoaei, and M. Shahabadi, "3-Coil orientation insensitive wireless power transfer for capsule endoscope," in Proc. 23rd Iranian Conf. Elect. Eng., May 2015, pp. 1249-1254.

[39] H. Lay et al., "In-vivo evaluation of microultrasound and thermometric capsule endoscopes," IEEE Trans. Biomed. Eng., to be published, doi: 10.1109/TBME.2018.2852715

[40] M. Acar, A. J. Annema, and B. Nauta, "Generalized design equations for Class-E power amplifiers with finite DC feed inductance," in Proc. Eur. Microw. Conf. (EuMC), Sep. 2006, pp. 1308-1311.

[41] H. Liu, Q. Shao, and X. Fang, "Modeling and optimization of class-E amplifier at subnominal condition in a wireless power transfer system for biomedical implants," IEEE Trans. Biomed. Circuits Syst., vol. 11, no. 1, pp. 35-43, Feb. 2017.
[42] P. Si and A. P. Hu, "Analyses of DC inductance used in ICPT power pick-Ups for maximum power transfer," in Proc. IEEE/PES Transmiss. Distrib. Conf. Expo. Asia Pacific, Aug. 2005, pp. 1-6.

[43] M. R. Basar, M. Y. Ahmad, J. Cho, and F. Ibrahim, "A wireles power transmission system for robotic capsule endoscopy: Design and optimization," in IEEE MTT-S Int. Microw. Symp. Dig., Dec. 2014, pp. 1-3.

[44] A.-M. Singeap, C. Stanciu, and A. Trifan, "Capsule endoscopy: The road ahead," World J. Gastroenterol., vol. 22, no. 1, pp. 369-378, Jan. 2016.

[45] S. R. Khan and G. S. Choi, "High-efficiency CMOS rectifier with minimized leakage and threshold cancellation features for low power bio-implants," Microelectron. J., vol. 66, pp. 67-75, Aug. 2017.

[46] M. M. Mohamed et al., "High-efficiency CMOS RF-to-DC rectifier based on dynamic threshold reduction technique for wireless charging applications," IEEE Access, vol. 6, pp. 46826-46832, 2018.

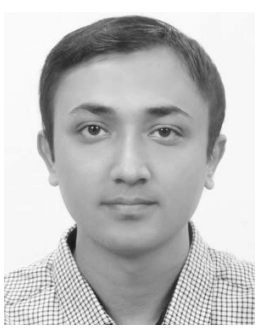

Sadeque Reza Khan received the B.Sc. degree in electronics and telecommunication engineering from the University of Liberal Arts Bangladesh, Dhaka, Bangladesh, in 2010, and the M.Tech. degree in very large scale integration design from the National Institute of Technology Karnataka, Mangalore, India, in 2014. He is currently pursuing the Ph.D. degree in electrical engineering at HeriotWatt University, Edinburgh, U.K.

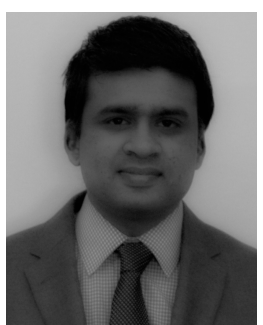

Sumanth Kumar Pavuluri received the Ph.D degree from Heriot-Watt University, Edinburgh, U.K., in 2011.

$\mathrm{He}$ is currently a Post-Doctoral Research Associate with Heriot-Watt University, where he is involved in microwave sensing, micromachined antennas, and microwave applicators for curing and heating applications.

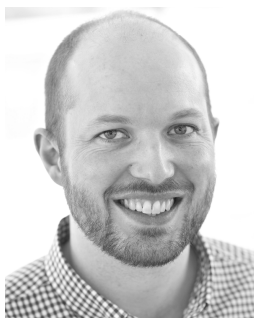

Gerard Cummins (SM'03) received the B.Eng. degree in microelectronic and electrical engineering from University College Cork, Cork, Ireland, and the $\mathrm{Ph} . \mathrm{D}$. degree in engineering from the University of Edinburgh, Edinburgh, U.K.

$\mathrm{He}$ is currently a Research Associate with the Institute of Sensors, Signals and Systems, HeriotWatt University, Edinburgh. His current research interests include microengineering, microsystems, and medical device design.

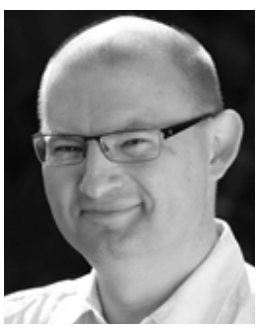

Marc P. Y. Desmulliez (SM'87) received the Ph.D. degree in optoelectronics from Heriot-Watt University, Edinburgh, U.K., in 1995.

$\mathrm{He}$ is currently a Professor of microsystems engineering with Heriot-Watt University, where he leads the Multimodal Sensing and Micromanipulation Research Group. 